Technical Note

\title{
On the Delayed Scaled Consensus Problems
}

\author{
Yilun Shang \\ School of Mathematical Sciences, Tongji University, Shanghai 200092, China; shylmath@hotmail.com \\ Academic Editors: Vicent Botti, Andrea Omicini, Stefano Mariani and Vicente Julian \\ Received: 28 May 2017; Accepted: 6 July 2017; Published: 11 July 2017
}

\begin{abstract}
In this note, we study the scaled consensus (tracking) problems, wherein all agents reach agreement, but with different assigned ratios in the asymptote. Based on the nearest neighbor-interaction rules, the scaled consensus processes are characterized with and without time delay. We consider both the signal transmission and signal processing delays and calculate the final scaled consensus values. When the underlying communication network contains a spanning tree, it is found that the scaled consensus can be achieved independent of the transmission delays while the specified consensus values in the asymptote depend on the initial history of the agents over a period of time. This phenomenon is in sharp contrast to the case of processing delays, where large delays are likely to jeopardize the consensus behavior, but the scaled consensus values once achieved are the same as the undelayed case.
\end{abstract}

Keywords: scaled consensus; delay; formation tracking; multi-agent system

\section{Introduction}

Multi-agent coordination of interconnected systems has found a diversity of applications in a number of fields, such as sensor networks, vehicle systems, social insects and cyber-physical systems. Consensus as a critical problem for multi-agent coordination aims to design appropriate protocols and strategies for reaching an agreement on a certain quantity of interest depending on the states of all agents. Different from traditional centralized controllers, the designed protocols take advantage of nearest-neighbor rules rendering the multi-agent systems in a distributed network framework governed by the graph Laplacians [1] since each agent can only interact with those within its local area due to limited communication capability. There has been an extensive literature on consensus problems using effective tools such as matrix theory, algebraic graph theory and system theory; see the recent surveys $[2,3]$ on this wide ranging topics.

In many practical applications, the states of all agents may achieve consensus on a common quantity, but of their own scales due to the constraints of physical environments. Examples include water distribution systems, compartmental mass-action systems [4] and multiscale coordination control between spacecraft and their simulating vehicles on the ground [5]. As an extension to standard consensus, Roy [6] recently introduced a novel notion of scaled consensus, which permits prescribed ratios among the final convergent values of all of the agents. The scaled consensus offers a less conservative framework, which can be specialized to achieve standard consensus (with all ratios being one), cluster consensus [7], where agents in a subnetwork share a common value while there is no agreement between different subnetworks, and bipartite consensus [8] or signed consensus [9] by adopting appropriate scales.

Scaled consensus has been studied for a fixed strongly-connected topology in $[6,10]$ and switching topologies in [11], where the agents are modeled by continuous-time single integrators. Scaled consensus can also be achieved through linear iterations $[12,13]$. However, time delay (especially distributed delay) has not been considered. Inspired by the stability analysis for delay systems in [14], we try to investigate the delayed scaled problems and distinguish between two main sources of time 
delays, to wit, signal transmission delays and signal processing delays. The contribution of this paper is two-fold. First, new scaled consensus protocols are proposed to accommodate discrete and distributed delays in networks containing spanning trees by extending the delayed complete consensus analysis in [14]. Second, for the undelayed scaled consensus process, we generalize some existing results on scaled consensus in $[6,10,11]$. In particular, we find that the ubiquitous spanning tree condition is both sufficient and necessary for scaled consensus, as well as for the related tracking formation problems on the scaled consensus manifold.

\section{Preliminaries}

The communication topology of a multi-agent system can often be characterized by a weighted directed graph [1] $\mathcal{G}=(\mathcal{V}, \mathcal{E}, \mathcal{A})$ with a node set $\mathcal{V}=\{1,2, \cdots, n\}$ representing $n$ agents, an edge set $\mathcal{E} \subseteq \mathcal{V} \times \mathcal{V}$ describing the information exchange among them and a nonnegative adjacency matrix $\mathcal{A}=\left(a_{i j}\right) \in \mathbb{R}^{n \times n}$. We assume $a_{i j}>0$ if and only if $(j, i) \in \mathcal{E}$, representing the information flow from agent $j$ to agent $i$. Because there are no self-loops in $\mathcal{G}$, we have $a_{i i}=0$ for all $i \in \mathcal{V}$. The degree of $i$ is defined as $d_{i}=\sum_{j=1}^{n} a_{i j}$. Given a sequence $i_{1}, i_{2} \cdots, i_{k}$ of distinct nodes, a directed path in $\mathcal{G}$ from $i_{1}$ to $i_{k}$ consists of a sequence of edges $\left(i_{j}, i_{j+1}\right) \in \mathcal{E}$ for $j=1, \cdots, k-1$. If there is a node (called root) in $\mathcal{G}$ from which all other nodes can be reached along directed paths, then we say that it has a spanning tree. Furthermore, $\mathcal{G}$ is said to be strongly connected if between any pair of distinct nodes $i, j$, there exists a directed path from $i$ to $j$. The graph Laplacian matrix of $\mathcal{A}, \mathcal{L}=\left(l_{i j}\right) \in \mathbb{R}^{n \times n}$, is defined by $l_{i i}=-\sum_{j \in \mathcal{V} \backslash\{i\}} l_{i j}=d_{i}$ and $l_{i j}=-a_{i j}$ for $i \neq j$. Clearly, $\mathcal{L} 1_{n}=0$, where $1_{n} \in \mathbb{R}^{n}$ represents the $n$-dimensional vector with elements being all ones. It is well known that $\mathcal{L}$ plays a significant role in the convergence analysis for consensus seeking [1]. By convention, the determinant, trace and adjugate of a square matrix are denoted by $\operatorname{det}(\cdot), \operatorname{tr}(\cdot)$ and $\operatorname{adj}(\cdot)$, respectively.

Here, we consider a group of $n$ agents, labeled from one to $n$, composing a fixed directed network $\mathcal{G}=(\mathcal{V}, \mathcal{E}, \mathcal{A})$. The dynamical behavior of each agent is described by a continuous-time system:

$$
\dot{x}_{i}(t)=u_{i}(t), \quad i \in \mathcal{V},
$$

where $x_{i}(t) \in \mathbb{R}$ is the information state and $u_{i}(t) \in \mathbb{R}$ is the control input of the agent $i$ at time $t$. In the vector form, we denote $x(t)=\left(x_{1}(t), \cdots, x_{n}(t)\right)^{\mathrm{T}} \in \mathbb{R}^{n}$ and let $x(0)=\left(x_{1}(0), \cdots, x_{n}(0)\right)^{\mathrm{T}} \in \mathbb{R}^{n}$ be the initial value. Given any scalar scale $\alpha_{i} \neq 0$ for the agent $i$, the system (1) is said to achieve scaled consensus to $\left(\alpha_{1}, \cdots, \alpha_{n}\right)$ if $\lim _{t \rightarrow \infty}\left(\alpha_{i} x_{i}(t)-\alpha_{j} x_{j}(t)\right)=0$ for all $i, j \in \mathcal{V}$ and all initial conditions $x(0)[6]$.

Remark 1. The notion of scaled consensus is a generalization of the standard consensus [2], cluster consensus [7] and bipartite consensus [8]. Note that the scales $\alpha_{i}$ associated with each individual $i$ can be positive or negative, adding to the flexibility of the consensus-seeking process. Scaled consensus can also be defined in terms of the global asymptotic stability of the manifold defined by the above condition $\lim _{t \rightarrow \infty}\left(\alpha_{i} x_{i}(t)-\alpha_{j} x_{j}(t)\right)=0$, both of which are known to be equivalent for linear processes [6].

\section{Undelayed Scaled Consensus Process}

In this section, we first consider the undelayed scaled consensus problem. Given the scales $\left(\alpha_{1}, \cdots, \alpha_{n}\right)$ with nonzero $\alpha_{i}$ 's, we apply the nearest-neighbor rules to propose the distributed strategy for agent $i$ as:

$$
u_{i}(t)=\operatorname{sgn}\left(\alpha_{i}\right) \sum_{j=1}^{n} a_{i j}\left(\alpha_{j} x_{j}(t)-\alpha_{i} x_{i}(t)\right)
$$

where $\operatorname{sgn}(\cdot)$ is the signum function. Let $\mathcal{D}=\operatorname{diag}\left(d_{1}, \cdots, d_{n}\right) \in \mathbb{R}^{n \times n}$ be the degree diagonal matrix. Then, we have $\mathcal{L}=\mathcal{D}-\mathcal{A}$. Moreover, for ease of presentation, we define $\alpha:=$ $\operatorname{diag}\left(\alpha_{1}, \cdots, \alpha_{n}\right) \in \mathbb{R}^{n \times n},|\alpha|:=\operatorname{diag}\left(\left|\alpha_{1}\right|, \cdots,\left|\alpha_{n}\right|\right) \in \mathbb{R}^{n \times n}, \alpha^{-1}:=\operatorname{diag}\left(\alpha_{1}^{-1}, \cdots, \alpha_{n}^{-1}\right) \in \mathbb{R}^{n \times n}$ 
and $\operatorname{sgn}(\alpha):=\operatorname{diag}\left(\operatorname{sgn}\left(\alpha_{1}\right), \cdots, \operatorname{sgn}\left(\alpha_{n}\right)\right) \in \mathbb{R}^{n \times n}$. With these notations, the switched multi-agent system (1) with Protocol (2) can be recast as:

$$
\dot{x}(t)=-\operatorname{sgn}(\alpha) \mathcal{L} \alpha x(t) .
$$

The following result gives a concise characterization for the scaled consensus process governed by (3).

Theorem 1. The multi-agent system (3) achieves scaled consensus to $\left(\alpha_{1}, \cdots, \alpha_{n}\right)$ if and only if $\mathcal{G}$ has a spanning tree.

Proof. Sufficiency: It follows from (3) that $x(t)=\exp (-\operatorname{sgn}(\alpha) \mathcal{L} \alpha t) x(0)$ for $t \geq 0$ using the matrix exponential. We first consider the matrix $|\alpha| \mathcal{L}$. Clearly, it has zero row sums, and hence, zero is always an eigenvalue of $|\alpha| \mathcal{L}$. By applying the Gershgorin disk theorem [15], we see that all eigenvalues of $|\alpha| \mathcal{L}$ are in the right half plane, and its nonzero eigenvalues have strictly positive real parts (cf. [16]). Since $\mathcal{G}$ has a spanning tree, $\lambda_{1}=0$ is a simple eigenvalue of the Laplacian $\mathcal{L}$, as well as of $|\alpha| \mathcal{L}$ recalling that $|\alpha|$ is a positive definite diagonal matrix [17]. Noting that $\alpha^{-1}|\alpha| \mathcal{L} \alpha=\operatorname{sgn}(\alpha) \mathcal{L} \alpha$, we see that the state matrix $-\operatorname{sgn}(\alpha) \mathcal{L} \alpha$ is similar to $-|\alpha| \mathcal{L}$. Hence, all eigenvalues of $-\operatorname{sgn}(\alpha) \mathcal{L} \alpha$, denoted by $\lambda_{1}=0, \lambda_{2}, \cdots, \lambda_{n}$, are in the left half plane, and $\lambda_{2}, \cdots, \lambda_{n}$ have strictly negative real parts.

It is direct to see that $\exp (-\operatorname{sgn}(\alpha) \mathcal{L} \alpha t)$ is a normal matrix, and its eigenvalues are $e^{\lambda_{1} t}, \cdots, e^{\lambda_{n} t}$. Therefore, $\exp (-\operatorname{sgn}(\alpha) \mathcal{L} \alpha t)$ possesses a complete set of eigenvectors, denoted by $\left\{v_{1}=\left(\alpha_{1}^{-1}, \cdots, \alpha_{n}^{-1}\right)^{\mathrm{T}}, v_{2}, \cdots, v_{n}\right\}$, which are exactly the corresponding eigenvectors of $-\operatorname{sgn}(\alpha) \mathcal{L} \alpha$ [15]. That is, $-\operatorname{sgn}(\alpha) \mathcal{L} \alpha v_{i}=\lambda_{i} v_{i}$ for $i=1, \cdots, n$. Let $\left\{w_{1}, \cdots, w_{n}\right\}$ be the set of linearly independent eigenvectors of $(\exp (-\operatorname{sgn}(\alpha) \mathcal{L} \alpha t))^{\mathrm{T}}$, such that $w_{i}^{\mathrm{T}} v_{j}=\delta_{i j}$, where $\delta_{i j}$ is the Kronecker delta for $i, j=1, \cdots, n$. Hence, $x(t)=\sum_{i=1}^{n}\left(w_{i}^{\mathrm{T}} x(t)\right) v_{i}$, and furthermore,

$$
\begin{aligned}
x(t) & =\exp (-\operatorname{sgn}(\alpha) \mathcal{L} \alpha t) x(0)=\sum_{i=1}^{n} w_{i}^{\mathrm{T}} \exp (-\operatorname{sgn}(\alpha) \mathcal{L} \alpha t) x(0) v_{i} \\
& =\sum_{i=1}^{n} e^{\lambda_{i} t} w_{i}^{\mathrm{T}} x(0) v_{i} \rightarrow w_{1}^{\mathrm{T}} x(0) v_{1}
\end{aligned}
$$

as $t$ tends to infinity. Recalling $v_{1}=\left(\alpha_{1}^{-1}, \cdots, \alpha_{n}^{-1}\right)^{\mathrm{T}}$, we see that the multi-agent system (3) achieves scaled consensus to $\left(\alpha_{1}, \cdots, \alpha_{n}\right)$.

Necessity: Suppose that $\mathcal{G}$ does not contain a spanning tree. Then, the zero eigenvalue is no longer a simple eigenvalue of $|\alpha| \mathcal{L}$ [17]. We consider the following two cases: (i) $\lambda_{i}=\lambda_{1}=0$ for some $i \neq 1$, and the geometric multiplicity of zero is equal to its algebraic multiplicity; and (ii) the geometric multiplicity of zero is less than the algebraic multiplicity.

In Case (i), say, $\lambda_{2}=\lambda_{1}=0$, we have $\dot{x}(t)=0$ for any $x(0) \in \operatorname{span}\left\{v_{2}\right\}$ by (3). However, for such initial values, scaled consensus to $\left(\alpha_{1}, \cdots, \alpha_{n}\right)$ is impossible because $v_{2} \notin \operatorname{span}\left\{v_{1}\right\}$. Next, we consider Case (ii). In this situation, the state matrix $-\operatorname{sgn}(\alpha) \mathcal{L} \alpha$ is similar to a Jordan matrix $J \in \mathbb{R}^{n \times n}$. Hence, $\exp (-\operatorname{sgn}(\alpha) \mathcal{L} \alpha t)=P^{-1} e^{J t} P$ for some invertible matrix $P$. For a $k \times k$ Jordan block $(k \geq 2)$, we have:

$$
\exp \left(\left(\begin{array}{cccc}
0 & 1 & \cdots & 0 \\
0 & 0 & \ddots & \vdots \\
\vdots & \vdots & \ddots & 1 \\
0 & 0 & \cdots & 0
\end{array}\right) t\right)=\left(\begin{array}{cccc}
1 & t & \cdots & \frac{t^{k-1}}{(k-1) !} \\
0 & 1 & \ddots & \vdots \\
\vdots & \vdots & \ddots & t \\
0 & 0 & \cdots & 1
\end{array}\right) \in \mathbb{R}^{k \times k}
$$

which is divergent with respect to $t$. Similar reasoning as (4) implies that $x_{i}(t) / x_{j}(t)$ does not approach $\alpha_{j} / \alpha_{i}$ for all $i, j$ and generic initial conditions $x(0)$. Thus, scaled consensus to $\left(\alpha_{1}, \cdots, \alpha_{n}\right)$ is not achieved, and this completes the proof of necessity. 
Remark 2. The scaled consensus protocol specified by (3) is first studied in [6]. It is proven that [6] if $G$ is strongly connected, then the scaled consensus can be reached. Theorem 1 improves the result by showing that a weaker spanning tree condition, actually, is not only sufficient, but necessary.

Remark 3. It is clear that the scaled consensus states are represented by $w_{1}^{\mathrm{T}} x(0) v_{1}=w_{1}^{\mathrm{T}} x(0)\left(\alpha_{1}^{-1}, \cdots, \alpha_{n}^{-1}\right)^{\mathrm{T}}$ and the scaled states $\alpha x(t) \rightarrow w_{1}^{\mathrm{T}} x(0) 1_{n}$, a common asymptote, as $t$ grows. In fact, the quantity $w_{1}^{\mathrm{T}} x(t)$ is conserved under the dynamics (3) since $\frac{d}{d t}\left(w_{1}^{\mathrm{T}} x(t)\right)=-w_{1}^{\mathrm{T}} \operatorname{sgn}(\alpha) \mathcal{L} \alpha x(t)=0$.

Similarly as in [6], we may extend Theorem 1 by considering a tracking dynamics on the scaled consensus manifold as:

$$
\dot{x}(t)=-\operatorname{sgn}(\alpha) \mathcal{L} \alpha x(t)+\dot{f}(t) \alpha^{-1} 1_{n}
$$

where the forcing input $f:[0,+\infty) \rightarrow \mathbb{R}$ is continuously differentiable. Setting $y(t)=x(t)-f(t) \alpha^{-1} 1_{n}$, we can prove the following result using the same argument as in Theorem 1.

Corollary 1. The multi-agent system (5) achieves scaled consensus to $\left(\alpha_{1}, \cdots, \alpha_{n}\right)$ if and only if $\mathcal{G}$ has a spanning tree. In particular, $x(t)$ converges to the time function $\left(w_{1}^{\mathrm{T}} x(0)+f(t)\right) v_{1}$ as $t$ grows.

\section{Scaled Consensus with Signal Transmission Delays}

Signal transmission delay, also known as coupling delay, can be introduce to the control scheme (1) by considering:

$$
u_{i}(t)=\operatorname{sgn}\left(\alpha_{i}\right) \sum_{j=1}^{n} a_{i j}\left(\alpha_{j} x_{j}(t-\tau)-\alpha_{i} x_{i}(t)\right),
$$

where $\tau \geq 0$ represents the time delay. Signal transmission delay often appears in networks of oscillators and has been investigated in, e.g., $[14,18]$ for standard consensus problems. In fact, (6) can be generalized to accommodate a history dependence over the interval $[t-\tau, t]$ instead of a discrete past time instant $t-\tau$ :

$$
u_{i}(t)=\operatorname{sgn}\left(\alpha_{i}\right) \sum_{j=1}^{n} a_{i j}\left(\int_{-\tau}^{0} \alpha_{j} x_{j}(t+s) d \eta(s)-\alpha_{i} x_{i}(t)\right)
$$

where $\eta:[-\tau, 0] \rightarrow \mathbb{R}$ is a function of bounded variation describing the distributed delays. Assume that $\eta$ is nondecreasing and appropriately normalized: $\int_{-\tau}^{0} d \eta(s)=1 . \eta$ can be viewed as a probability distribution and admits scaled consensus solutions. In particular, when $\eta$ is a Heaviside step function, the control input (7) readily reduces to (6).

The switched multi-agent system (1) with Protocol (7) can be recast in a compact form as:

$$
\dot{x}(t)=-\operatorname{sgn}(\alpha) \mathcal{D} \alpha x(t)+\operatorname{sgn}(\alpha) \mathcal{A} \alpha \int_{-\tau}^{0} x(t+s) d \eta(s) .
$$

The formulation $x(t)=e^{\omega t} v\left(v \in \mathbb{R}^{n}\right)$ yields the characteristic equation in $\omega \in \mathbb{C}$ as:

$$
\chi(\omega):=\operatorname{det}\left(\omega I_{n}+\operatorname{sgn}(\alpha) \mathcal{D} \alpha-G(\omega) \operatorname{sgn}(\alpha) \mathcal{A} \alpha\right)=0,
$$

where $I_{n} \in \mathbb{R}^{n \times n}$ is the $n$-dimensional identity matrix and $G(\omega)=\int_{-\tau}^{0} e^{\omega s} d \eta(s)$. Let $\bar{\tau}:=-\int_{-\tau}^{0} s d \eta(s) \geq 0$ represent the mean delay. It is clear that $G(0)=1$ and $G^{\prime}(0)=-\bar{\tau}$. Moreover, zero is always a characteristic value of (9) since $\chi(0)=\operatorname{det}(\operatorname{sgn}(\alpha)) \operatorname{det}(\mathcal{L}) \operatorname{det}(\alpha)=0$.

Our main result in this section shows that the stability of scaled consensus is independent of the transmission delays, generalizing the stability result of standard consensus [14] with all $\alpha_{i}$ being one.

Theorem 2. If $\mathcal{G}$ has a spanning tree, the multi-agent system (8) achieves scaled consensus to $\left(\alpha_{1}, \cdots, \alpha_{n}\right)$. 
Proof. We first show that all nonzero characteristic values of (9) have negative real parts. In fact, assume that $\chi(\omega)=0$ and $\operatorname{Re}(\omega) \geq 0$. It follows from (9) that $-\omega$ is an eigenvalue of $\operatorname{sgn}(\alpha) \mathcal{D} \alpha-$ $G(\omega) \operatorname{sgn}(\alpha) \mathcal{A} \alpha$. Note that the matrix $\operatorname{sgn}(\alpha) \mathcal{D} \alpha-G(\omega) \operatorname{sgn}(\alpha) \mathcal{A} \alpha$ is similar to $|\alpha| \mathcal{D}-G(\omega)|\alpha| \mathcal{A}$. In light of the Gershgorin disk theorem, the eigenvalues of $\operatorname{sgn}(\alpha) \mathcal{D} \alpha-G(\omega) \operatorname{sgn}(\alpha) \mathcal{A} \alpha$ must lie in the union of $n$ disks centered at $\left|\alpha_{i}\right| d_{i}$ with radius $|G(\omega)| \cdot\left|\alpha_{i}\right| \cdot \sum_{j=1}^{n} a_{i j} \leq\left|\alpha_{i}\right| d_{i} \cdot \int_{-\tau}^{0} d \eta(s) \leq\left|\alpha_{i}\right| d_{i}$ for $i=1, \cdots, n$. This implies that the nonzero characteristic values of (9) are contained in the set $\{\omega \in \mathbb{C}: \operatorname{Re}(\omega)>0\} \cup\{0\}$, and thus, $\operatorname{Re}(-\omega) \leq 0$. Accordingly, we have $\omega=0$, which completes the proof of the claim.

Next, we show that zero is a simple characteristic value of (9). It follows from (9) and some basic algebras [15] that:

$$
\begin{aligned}
\chi^{\prime}(0) & =\operatorname{tr}\left(\operatorname{adj}(\operatorname{sgn}(\alpha) \mathcal{L} \alpha) \cdot\left(I_{n}+\bar{\tau} \operatorname{sgn}(\alpha) \mathcal{A} \alpha\right)\right) \\
& =\operatorname{tr}\left(\operatorname{adj}(\operatorname{sgn}(\alpha) \mathcal{L} \alpha) \cdot\left(I_{n}+\bar{\tau} \operatorname{sgn}(\alpha)(\mathcal{D}-\mathcal{L}) \alpha\right)\right) \\
& =\operatorname{tr}\left(\operatorname{adj}(\operatorname{sgn}(\alpha) \mathcal{L} \alpha) \cdot\left(I_{n}+\bar{\tau} \operatorname{sgn}(\alpha) \mathcal{D} \alpha\right)\right)
\end{aligned}
$$

where in the last equality, we note that $\operatorname{adj}(\operatorname{sgn}(\alpha) \mathcal{L} \alpha) \cdot \operatorname{sgn}(\alpha) \mathcal{L} \alpha=\operatorname{det}(\operatorname{sgn}(\alpha) \mathcal{L} \alpha) I_{n}=$ $\operatorname{det}(\operatorname{sgn}(\alpha)) \operatorname{det}(\mathcal{L}) \operatorname{det}(\alpha) I_{n}=0$. Let $\tilde{\ell}_{i i}$ represent the diagonal entries of $\operatorname{adj}(\operatorname{sgn}(\alpha) \mathcal{L} \alpha)$. Then:

$$
\chi^{\prime}(0)=\sum_{i=1}^{n} \tilde{\ell}_{i i}\left(1+\bar{\tau}\left|\alpha_{i}\right| d_{i}\right)
$$

As in Theorem 1, we know that zero is a simple eigenvalue of $|\alpha| \mathcal{L}$ since $\mathcal{G}$ contains a spanning tree. Since zero is also a simple eigenvalue of $\operatorname{sgn}(\alpha) \mathcal{L} \alpha$ due to similarity, the matrix $\operatorname{sgn}(\alpha) \mathcal{L} \alpha$ has rank $n-1$ and a one-dimensional kernel spanned by $v_{1}=\left(\alpha_{1}^{-1}, \cdots, \alpha_{n}^{-1}\right)^{\mathrm{T}}$. Therefore, $\operatorname{adj}(\operatorname{sgn}(\alpha) \mathcal{L} \alpha) \neq 0$. Moreover, we have $\operatorname{sgn}(\alpha) \mathcal{L} \alpha \cdot \operatorname{adj}(\operatorname{sgn}(\alpha) \mathcal{L} \alpha)=\operatorname{det}(\operatorname{sgn}(\alpha) \mathcal{L} \alpha) I_{n}=0$, which implies that the columns of $\operatorname{adj}(\operatorname{sgn}(\alpha) \mathcal{L} \alpha)$ belong to the kernel of $\operatorname{sgn}(\alpha) \mathcal{L} \alpha$. Accordingly, $\operatorname{adj}(\operatorname{sgn}(\alpha) \mathcal{L} \alpha)=v_{1} u$ for some row vector $u=\left(u_{1}, \cdots, u_{n}\right)$. Analogously, the equality $\operatorname{adj}(\operatorname{sgn}(\alpha) \mathcal{L} \alpha) \cdot \operatorname{sgn}(\alpha) \mathcal{L} \alpha=0$ implies that the rows of $\operatorname{adj}(\operatorname{sgn}(\alpha) \mathcal{L} \alpha)$ belong to $\operatorname{span}\left\{w_{1}\right\}$, where $w_{1} \in \mathbb{R}^{n}$ is defined in the proof of Theorem 1 . Hence, for each $1 \leq i \leq n$, there exists some $c_{i} \in \mathbb{R}$ such that:

$$
\left(\frac{u_{1}}{\alpha_{i}}, \cdots, \frac{u_{n}}{\alpha_{i}}\right)=c_{i} w_{1}^{\mathrm{T}}
$$

If $c_{i}=0$, then the $i$-th row in the matrix (11) becomes zero, and $u_{1}=\cdots=u_{n}=0$. This indicates $\operatorname{adj}(\operatorname{sgn}(\alpha) \mathcal{L} \alpha)=0$, which is a contradiction. Therefore, $c_{i} \neq 0$ for all $i$. We can show in the same way that $u_{i} \neq 0$ for all $i$. Therefore, there exists some $k \neq 0$ such that $c_{i}=\frac{k}{\alpha_{i}}$ and $\tilde{\ell}_{i i}=\frac{k w_{1 i}}{\alpha_{i}}$ for all $1 \leq i \leq n$, where we write $w_{1}=\left(w_{11}, \cdots, w_{1 n}\right)^{\mathrm{T}}$. In view of $(10)$, we have:

$$
\chi^{\prime}(0)=k \sum_{i=1}^{n} \frac{w_{1 i}}{\alpha_{i}}\left(1+\bar{\tau}\left|\alpha_{i}\right| d_{i}\right)
$$

By definition, $w_{1}^{\mathrm{T}} \operatorname{sgn}(\alpha) \mathcal{L} \alpha=0$. Writing $w_{1}^{\mathrm{T}} \operatorname{sgn}(\alpha):=\tilde{w}_{1}^{\mathrm{T}}=\left(\tilde{w}_{11}, \cdots, \tilde{w}_{1 n}\right)$, we obtain $\tilde{w}_{1}^{\mathrm{T}} \mathcal{L}=0$. For a sufficiently small $\varepsilon>0, I_{n}-\varepsilon \mathcal{L}$ is a stochastic matrix, and $\tilde{w}_{1}^{\mathrm{T}}(I-\varepsilon \mathcal{L})=\tilde{w}_{1}^{\mathrm{T}}$. Based on the Perron-Frobenius theory, we know that $\tilde{w}_{1}^{\mathrm{T}}$ (under an appropriate normalization) is the stationary distribution of the Markov chain described by the stochastic matrix. Hence, $\tilde{w}_{1}^{\mathrm{T}} \neq 0$ and has non-negative entries. Noting that $\frac{w_{1 i}}{\alpha_{i}}=\frac{\tilde{w}_{1 i} \operatorname{sgn}\left(\alpha_{i}\right)}{\alpha_{i}}$ and $1+\bar{\tau}\left|\alpha_{i}\right| d_{i}>0$ for all $i$, we see from (12) that $\chi^{\prime}(0) \neq 0$. Consequently, zero is a simple characteristic value of (9).

Since all solutions of (8) involve a factor $e^{\omega t}$ with $\omega$ being a root of the characteristic Equation (9). Note that the root $\omega=0$ corresponds to the kernel of $\operatorname{sgn}(\alpha) \mathcal{L} \alpha$, i.e., $\operatorname{span}\left\{v_{1}\right\}$. Moreover, the dynamics 
on the subspace $\operatorname{span}\left\{v_{1}\right\}$ is constant, to wit, $\dot{x}(t) \equiv 0$. Combining the above discussions, we are led to the conclusion that:

$$
\lim _{t \rightarrow \infty} x(t)=c v_{1}=c\left(\alpha_{1}^{-1}, \cdots, \alpha_{n}^{-1}\right)^{\mathrm{T}}
$$

for some $c \in \mathbb{R}$. The proof is complete.

Remark 4. The quantity $c$ in (13) can be determined. In fact, noting that $w_{1}^{\mathrm{T}} \operatorname{sgn}(\alpha) \mathcal{L} \alpha=0$, we obtain from (8) that:

$$
\begin{aligned}
& \frac{d}{d t}\left(w_{1}^{\mathrm{T}}\left(x(t)+\operatorname{sgn}(\alpha) \mathcal{D} \alpha \int_{-\tau}^{0} \int_{t+s}^{t} x\left(t^{\prime}\right) d t^{\prime} d \eta(s)\right)\right) \\
= & w_{1}^{\mathrm{T}} \dot{x}(t)+w_{1}^{\mathrm{T}} \operatorname{sgn}(\alpha) \mathcal{D} \alpha \int_{-\tau}^{0}(x(t)-x(t+s)) d \eta(s) \\
= & w_{1}^{\mathrm{T}} \operatorname{sgn}(\alpha) \mathcal{A} \alpha \int_{-\tau}^{0} x(t+s) d \eta(s)-w_{1}^{\mathrm{T}} \operatorname{sgn}(\alpha) \mathcal{D} \alpha \int_{-\tau}^{0} x(t+s) d \eta(s)=0 .
\end{aligned}
$$

This means that the quantity $w_{1}^{\mathrm{T}}\left(x(t)+\operatorname{sgn}(\alpha) \mathcal{D} \alpha \int_{-\tau}^{0} \int_{t+s}^{t} x\left(t^{\prime}\right) d t^{\prime} d \eta(s)\right)$ remains unchanged with respect to time. By setting $t \rightarrow \infty$, we have:

$$
\begin{aligned}
& w_{1}^{\mathrm{T}}\left(x(0)+\operatorname{sgn}(\alpha) \mathcal{D} \alpha \int_{-\tau}^{0} \int_{s}^{0} x(t) d t d \eta(s)\right) \\
= & w_{1}^{\mathrm{T}}\left(c v_{1}+\operatorname{sgn}(\alpha) \mathcal{D} \alpha \int_{-\tau}^{0} \int_{s}^{0} c v_{1} d t d \eta(s)\right) \\
= & c-c w_{1}^{\mathrm{T}} \operatorname{sgn}(\alpha) \mathcal{D} \alpha v_{1} \int_{-\tau}^{0} s d \eta(s) \\
= & c\left(1+\bar{\tau} w_{1}^{\mathrm{T}} \operatorname{sgn}(\alpha) \mathcal{D} 1_{n}\right) .
\end{aligned}
$$

Hence, we have:

$$
c=\frac{1}{1+\bar{\tau} w_{1}^{\mathrm{T}} \operatorname{sgn}(\alpha) \mathcal{D} 1_{n}} w_{1}^{\mathrm{T}}\left(x(0)+\operatorname{sgn}(\alpha) \mathcal{D} \alpha \int_{-\tau}^{0} \int_{s}^{0} x(t) d t d \eta(s)\right) .
$$

In the case of no delay, i.e., $\bar{\tau}=0$, (14) reduces to $c=w_{1}^{\mathrm{T}} x(0)$, which agrees with Equation (4) in [6]. In the case of discrete delay delineated by (6), we have:

$$
c=\frac{1}{1+\tau w_{1}^{\mathrm{T}} \operatorname{sgn}(\alpha) \mathcal{D} 1_{n}} w_{1}^{\mathrm{T}}\left(x(0)+\operatorname{sgn}(\alpha) \mathcal{D} \alpha \int_{-\tau}^{0} x(t) d t\right) .
$$

Remark 5. From (14), we observe that the scaled consensus values rely on the initial history of the agents' state $x$ over the interval $[-\tau, 0]$ and that the scaled consensus is guaranteed independent of the magnitude of transmission delays. An important implication is that, in the event of additive measurement noise with zero temporal mean, the delayed system may have better performance by choosing a suitable $\tau$ to reduce the noise effect by averaging over $[-\tau, 0]$ via (14). In [18], a similar procedure for delayed standard consensus problems has been discussed.

Finally, we consider a tracking dynamics on the scaled consensus manifold as:

$$
\dot{x}(t)=-\operatorname{sgn}(\alpha) \mathcal{D} \alpha x(t)+\operatorname{sgn}(\alpha) \mathcal{A} \alpha \int_{-\tau}^{0} x(t+s) d \eta(s)+\dot{f}(t) \alpha^{-1} 1_{n},
$$

where the forcing input $f$ is defined as in Section 3. We can similarly derive the following useful corollary. 
Corollary 2. If $\mathcal{G}$ has a spanning tree, then the multi-agent system (15) achieves scaled consensus to $\left(\alpha_{1}, \cdots, \alpha_{n}\right)$. In particular, $x(t)$ converges to the time function $(c+f(t)) v_{1}$ as $t$ grows, where $c$ is given by (14).

\section{Scaled Consensus with Signal Processing Delays}

In this section, we examine the signal processing delay, which is also known as internal delay. Signal processing delay has been much studied in the literature of consensus problems; see, e.g., [1-3]. In parallel with (6), the scaled consensus control scheme can be designed as:

$$
u_{i}(t)=\operatorname{sgn}\left(\alpha_{i}\right) \sum_{j=1}^{n} a_{i j}\left(\alpha_{j} x_{j}(t-\tau)-\alpha_{i} x_{i}(t-\tau)\right)
$$

where $\tau \geq 0$ indicates the time delay. The distributed-delay version is:

$$
u_{i}(t)=\operatorname{sgn}\left(\alpha_{i}\right) \sum_{j=1}^{n} a_{i j} \int_{-\tau}^{0}\left(\alpha_{j} x_{j}(t+s)-\alpha_{i} x_{i}(t+s)\right) d \eta(s),
$$

where $\eta:[-\tau, 0] \rightarrow \mathbb{R}$ is a function of bounded variation describing the distributed delays. In analogy to (8), the switched multi-agent system (1) with protocol (17) can be written as:

$$
\dot{x}(t)=-\operatorname{sgn}(\alpha) \mathcal{L} \alpha \int_{-\tau}^{0} x(t+s) d \eta(s) .
$$

The characteristic equation is given by:

$$
\chi(\omega):=\operatorname{det}\left(\omega I_{n}+G(\omega) \operatorname{sgn}(\alpha) \mathcal{L} \alpha\right)=0,
$$

where $\omega \in \mathbb{C}$ and $G(\omega)=\int_{-\tau}^{0} e^{\omega s} d \eta(s)$. Similarly as the transmission delay case, we have $G(0)=1$, $\chi(0)=0$ and $G^{\prime}(0)=-\bar{\tau}:=\int_{-\tau}^{0} s d \eta(s)$.

Here, we will only study the system (1) with discrete delay (16), which corresponds to a special form $G(\omega)=e^{-\omega \tau}$. We assume the following detailed balanced condition.

Assumption 1. $\left|\alpha_{i}\right| a_{i j}=\left|\alpha_{j}\right| a_{j i}$ holds for all $i, j=1, \cdots, n$.

The detailed balanced condition has proven to be instrumental in studying coupled dynamics; see, e.g., $[19,20]$ for details. Our main result in this section reads as follows.

Theorem 3. Suppose that $\mathcal{G}$ has a spanning tree and that Assumption 1 holds. The multi-agent system (1) with Protocol (16) achieves scaled consensus to $\left(\alpha_{1}, \cdots, \alpha_{n}\right)$ if and only if $0 \leq \tau<\frac{\pi}{2 \max _{1 \leq i \leq n} \lambda_{i}}$, where $\left\{\lambda_{i}\right\}_{i=1}^{n}$ are the eigenvalues of $|\alpha| \mathcal{L}$.

Proof. Note that $|\alpha| \mathcal{L}$ is symmetric and positive semidefinite under Assumption 1. Let $\lambda_{1}=0$. The spanning tree condition implies $\lambda_{i}>0$ for $i=2, \cdots, n$.

We first show that zero is a simple characteristic value of (19). Indeed, we already see that $\chi(0)=0$. Arguing similarly as in Theorem 2, we have:

$$
\begin{aligned}
\chi^{\prime}(0) & =\operatorname{tr}\left(\operatorname{adj}(\operatorname{sgn}(\alpha) \mathcal{L} \alpha) \cdot\left(I_{n}-\tau \operatorname{sgn}(\alpha) \mathcal{L} \alpha\right)\right) \\
& =\operatorname{tr}(\operatorname{adj}(\operatorname{sgn}(\alpha) \mathcal{L} \alpha))=k \sum_{i=1}^{n} \frac{w_{1 i}}{\alpha_{i}} \neq 0,
\end{aligned}
$$

where $k \neq 0$ and $w_{1}=\left(w_{11}, \cdots, w_{1 n}\right)^{\mathrm{T}}$ are interpreted in the same way as in (12). This means zero is a simple root of (19). 
Next, we show that $0<\lambda_{i} \tau<\frac{\pi}{2}$ for all $i \geq 2$ if and only if all nonzero characteristic values of (19) have negative real parts. Suppose that $\chi(\omega)=0$ for some $\omega \in \mathbb{C}$. By (19), we know that $\frac{-\omega}{G(\omega)}=-\omega e^{\omega \tau}$ is an eigenvalue of $|\alpha| \mathcal{L}$. That is, $\omega e^{\omega \tau}=\lambda_{i}(i=2, \cdots, n)$. Under Assumption 1 , Theorem 1 in [21] implies that $\operatorname{Re}(\omega)<0$ for all solutions $\omega$ of the above system if and only if $0<\lambda_{i} \tau<\frac{\pi}{2}$ for $i=2, \cdots, n$.

It is easy to see that the sufficiency of Theorem 3 holds by the same argument at the end of Theorem 2 . The necessity of Theorem 3 follows similarly from the necessity proof of Theorem 1.

Remark 6. It follows from the Gershgorin disk theorem that $0 \leq \lambda_{i} \leq 2 \max _{1 \leq i \leq n}\left|\alpha_{i}\right| d_{i}$. Therefore, the eigenvalue condition in Theorem 3 can be replaced by a more geometric (sufficient) condition $0 \leq \tau<$ $\frac{\pi}{4 \max _{1 \leq i \leq n}\left|\alpha_{i}\right| d_{i}}$. We mention that a deep investigation regarding the conditions guaranteeing $\operatorname{Re}\left(\lambda_{i}\right)<0(i \geq 2)$ would be critical for tackling the general system (18).

Remark 7. Since $\frac{d}{d t}\left(w_{1}^{\mathrm{T}} x(t)\right)=-w_{1}^{\mathrm{T}} \operatorname{sgn}(\alpha) \mathcal{L} \alpha x(t-\tau)=0$, the quantity $w_{1}^{\mathrm{T}} x(t)$ is conserved with respect to time $t$. Thus, setting $\lim _{t \rightarrow \infty} x(t)=c v_{1}$, we have $w_{1}^{\mathrm{T}} x(0)=c w_{1}^{\mathrm{T}} v_{1}=c$, which is the same as the undelayed multi-agent system (3).

Remark 8. From Theorem 3, we find that introducing signal processing delay may prohibit the scaled consensus process, while the scaled consensus values are independent of the magnitude of the delay once scaled consensus is achieved, which is in sharp contrast to the effect of transmission delay.

By considering the following tracking dynamics:

$$
\dot{x}(t)=-\operatorname{sgn}(\alpha) \mathcal{L} \alpha x(t-\tau)+\dot{f}(t) \alpha^{-1} 1_{n}
$$

we can similarly derive the following corollary.

Corollary 3. Suppose that $\mathcal{G}$ has a spanning tree and Assumption 1 holds. The multi-agent system (20) achieves scaled consensus to $\left(\alpha_{1}, \cdots, \alpha_{n}\right)$ if and only if $0 \leq \tau<\frac{\pi}{2 \max _{1 \leq i \leq n} \lambda_{i}}$, where $\left\{\lambda_{i}\right\}_{i=1}^{n}$ are the eigenvalues of $|\alpha| \mathcal{L}$. In particular, $x(t)$ converges to the time function $\left(w_{1}^{\mathrm{T}} x(0)+f(t)\right) v_{1}$ as $t$ grows.

An example of delayed scaled consensus over a graph $\mathcal{G}$ of $n=4$ agents with $\alpha=\operatorname{diag}(1,-1,1,2)$ and $\mathcal{A}=\left(\begin{array}{llll}0 & 1 & 1 & 0 \\ 1 & 0 & 1 & 2 \\ 1 & 1 & 0 & 0 \\ 0 & 1 & 0 & 0\end{array}\right)$ is shown in Figure 1. Here, we take $\tau=0.2$ and $x(-\tau)=(3,-2,-1,1)^{\mathrm{T}}$. It is direct to check that the conditions of Theorems 2 and 3 are satisfied. As one would expect, the scaled consensus to $(1,-1,1,2)$ has been achieved under both signal transmission delays (Figure 2a) and signal processing delays (Figure $2 b$ ).

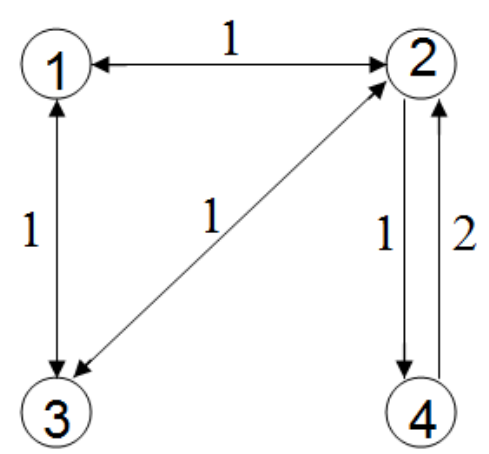

Figure 1. A communication network $\mathcal{G}$ over $n=4$ agents. 


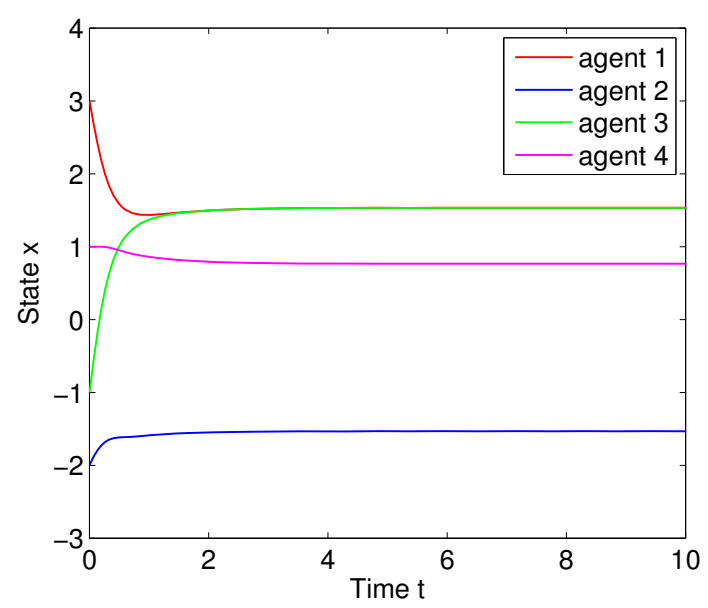

(a)Transmission delay

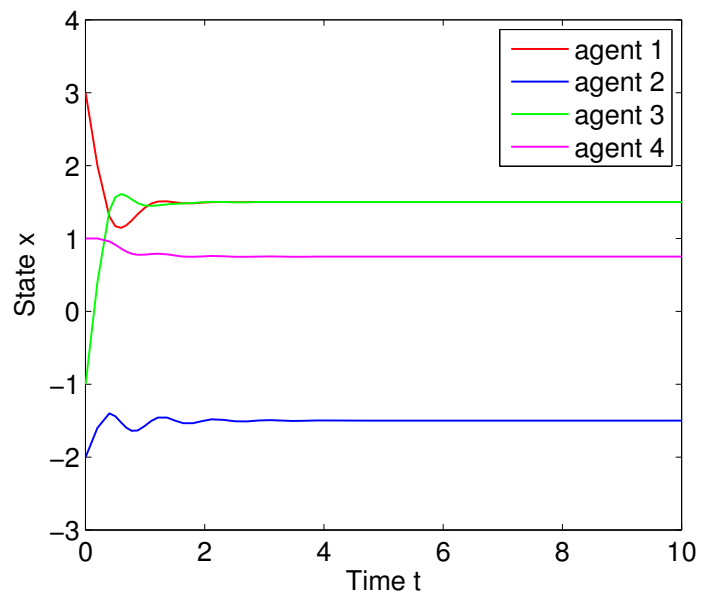

(b)Processing delay

Figure 2. State evolution of agents under (a) signal transmission delays for system (1) with (6) and (b) signal processing delays for system (1) with (16).

\section{Conclusions}

Scaled consensus problems have found diverse applications in cooperative tasks in practical multi-agent systems. This paper discusses the stability of scaled consensus problems with and without time delays. We distinguish between signal transmission delays and signal processing delays by deriving sufficient and necessary scaled consensus conditions and calculating the final scaled consensus values in each case. These two types of delays are shown to possess distinct features on the scaled consensus processes, as well as the scaled consensus tracking on associated manifolds.

The scales encoded in $\alpha$ in this paper are assumed to be constant. It would be interesting to explore time-varying scales $\alpha=\alpha(t)$ (leading to a non-autonomous linear system) in the delayed system framework. It is noteworthy that our results assume a fixed communication topology and homogeneous agents. Therefore, delayed scaled consensus processes with switching/uncertain topologies and heterogeneously motivated agents (e.g., [22]) can be meaningful subjects of future work.

Acknowledgments: The author would like to thank the anonymous referees for their valuable suggestions that improved the presentation of the paper. The work is funded in part by the National Natural Science Foundation of China (11505127), the Shanghai Pujiang Program (15PJ1408300) and the Program for Young Excellent Talents in Tongji University (2014KJ036).

Conflicts of Interest: The author declares no conflict of interest. 


\section{References}

1. Mesbahi, M.; Egerstedt, M. Graph Theoretic Methods in Multiagent Networks; Princeton University Press: Princeton, NJ, USA, 2010.

2. Olfati-Saber, R.; Fax, J.A.; Murray, R.M. Consensus and cooperation in networked multi-agent systems. Proc. IEEE 2007, 95, 215-233.

3. Cao, Y.; Yu, W.; Ren, W.; Chen, G. An overview of recent progress in the study of distributed multi-agent coordination. IEEE Trans. Ind. Inf. 2013, 9, 427-438.

4. Haddad, W.M.; Chellaboina, V.; Hui, Q. Nonnegative and Compartmental Dynamical Systems; Princeton University Press: Princeton, NJ, USA, 2010.

5. Guglieri, G.; Maroglio, F.; Pellegrino, P.; Torre, L. Design and development of guidance navigation and control algorithms for spacecraft rendezvous and docking experimentation. Acta Astronaut. 2014, 94, 395-408.

6. Roy, S. Scaled consensus. Automatica 2015, 51, 259-262.

7. Shang, Y. A combinatorial necessary and sufficient condition for cluster consensus. Neurocomputing 2016, 216, 611-616.

8. Altafini, C. Consensus problems on networks with antagonistic interactions. IEEE Trans. Autom. Control 2013, 58, 935-946.

9. Li, J.; Dong, W.; Xiao, H. Signed consensus problems on networks of agents with fixed and switching topologies. Int. J. Control 2017, 90, 148-160.

10. Meng, D.; Jia, Y. Robust consensus algorithms for multiscale coordination control of multivehicle systems with disturbances. IEEE Trans. Ind. Electron. 2016, 63, 1107-1119.

11. Meng, D.; Jia, Y. Scaled consensus problems on switching networks. IEEE Trans. Autom. Control 2016, 61, 1664-1669.

12. Hou, B.; Sun, F.; Li, H.; Chen, Y.; Liu, G. Scaled cluster consensus of discrete-time multi-agent systems with general directed topologies. Int. J. Control 2016, 47, 3839-3845.

13. Shang, Y. Finite-time scaled consensus through parametric linear iterations. Int. J. Syst. Sci. 2017, 48, 2033-2040.

14. Atay, F.M. On the duality between consensus problems and Markov processes, with application to delay systems. Markov Process. Relat. Field. 2016, 22, 537-553.

15. Horn, R.A.; Johnson, C.R. Matrix Analysis; Cambridge University Press: Cambridge, UK, 2012.

16. Ren, W.; Beard, R.W. Consensus seeking in multiagent systems under dynamically changing interaction topologies. IEEE Trans. Autom. Control 2005, 50, 655-661.

17. Merris, R. Laplacian matrices of graphs: A survey. Linear Algebra Appl. 1994, 197-198, 143-176.

18. Atay, F.M. The consensus problem in networks with transmission delays. Phil. Trans. R. Soc. A 2013, 371, doi:10.1098/rsta.2012.0460.

19. Haken, H. Synergetics; Springer: Berlin, Germany, 1978.

20. Chu, T.; Zhang, C.; Zhang, Z. Necessary and sufficient condition for absolute stability of normal neural networks. Neural Netw. 2003, 16, 1223-1227.

21. Hayes, H.D. Roots of the transcendental equation associated with a certain differential difference equation. J. Lond. Math. Soc. 1950, 25, 226-232.

22. Shang, Y. Consensus in averager-copier-voter networks of moving dynamical agents. Chaos 2017, 27, doi:10.1063/1.4976959.

(C) 2017 by the author. Licensee MDPI, Basel, Switzerland. This article is an open access article distributed under the terms and conditions of the Creative Commons Attribution (CC BY) license (http://creativecommons.org/licenses/by/4.0/). 